\title{
Dynamic expression of the pro-dopaminergic transcription factors Pax6 and DIx2 during postnatal olfactory bulb neurogenesis
}

\section{Antoine de Chevigny ${ }^{1,2 *}$, Nathalie Core ${ }^{1,2}$, Philipp Follert ${ }^{1,2}$, Stefan Wild $^{3}$, Andreas Bosio $^{3}$, Kazuaki Yoshikawa ${ }^{4}$, Harold Cremer ${ }^{1,2}$ and Christophe Beclin ${ }^{1,2 *}$}

\author{
${ }^{1}$ IBDML, Institut de Biologie du Développement de Marseille-Luminy, CNRS/Université de la Méditerranée, Campus de Luminy, Marseille, France \\ ${ }^{2}$ CNRS, UMR7288, Marseille, France \\ ${ }^{3}$ Miltenyi Biotec GmbH, Friedrich-Ebert-Straße, Bergisch Gladbach, Germany \\ ${ }^{4}$ Laboratory of Regulation of Neuronal Development, Institute for Protein Research Osaka University, Yamadaoka, Suita, Osaka, Japan
}

Edited by:

Afsaneh Gaillard, INSERM U1084,

France

Reviewed by:

Francis G. Szele, Oxford University, UK

Shaoyu Ge, Suny Stony Brook, USA

Bhaskar Saha, Institut de

Physiologie et Biologie Cellulaires,

France

*Correspondence:

Antoine de Chevigny and Christophe Beclin, IBDML, Institut de Biologie

du Développement de

Marseille-Luminy, Campus de

Luminy, 13288 Marseille cedex 9,

Marseille, France.

e-mail: antoine.de-chevigny@

ibdml.univmed.fr;

christophe.beclin@ibdml.univmed.fr
Olfactory bulb $(\mathrm{OB})$ neurogenesis generates neurons that use GABA or dopamine as their neurotransmitters throughout life. Regionalized stem cell populations in the periventricular zone (PVZ) of the lateral ventricles (LVs) have been shown to be at the basis of neuronal diversity in the system. For example dopaminergic neurons arise predominantly from neural stem cells (NSCs) residing in the dorsal PVZ and depend on the expression of the transcription factors Pax 6 and Dlx2 for their specification. In addition, Dlx2 is required for neurogenesis in general. Using targeted in vivo electroporation combined with immuno-fluorescence imaging and microarray analysis, we provide here detailed spatial and temporal expression data with cellular resolution in this system. We find that all along the neurogenic process Pax6 expression remains restricted to the dorsal $P V Z$, whereas nearly all neuroblasts express Dlx2, including those of the dorsal lineage, which are switched on for Dlx2 when they enter the rostral migratory stream (RMS). These data allow to explain and precise the functions of these two genes in postnatal OB neurogenesis.

Keywords: postnatal neurogenesis, DIx2, Pax6, in vivo electroporation, SVZ, olfactory bulb

\section{INTRODUCTION}

Neurogenesis persists in the postnatal and adult mouse olfactory bulb $(\mathrm{OB})$. In this system, neuroblasts are generated in the periventricular zone (PVZ) of the lateral ventricles (LVs). After a phase of amplification they migrate along the rostral migratory stream (RMS) to differentiate into $\mathrm{OB}$ interneurons that play a major role in olfactory function (Lledo et al., 2006). The newly generated interneurons in the $\mathrm{OB}$ present a large diversity of phenotypes. For example, the superficial region of the OB contains many dopaminergic neurons whereas neurons located in the deeper layers are purely GABAergic (Alvarez-Buylla and GarciaVerdugo, 2002; Lledo et al., 2008). It has been shown that this phenotypic diversity relies on the spatial distribution of the corresponding neural stem cells (NSCs) in the germinal zone, the PVZ (Merkle et al., 2007). The molecular determinants of this mosaic organization in the SVZ are still poorly understood and only start to be investigated. For example, it has been recently shown that Shh signaling in the most ventral part of PVZ controls differentiation of a particular $\mathrm{OB}$ neuronal subtype that is characterized by calbindin expression (Ihrie et al., 2011).

Dopaminergic neurons of the $\mathrm{OB}$ represent a population of particular interest for cell replacement strategies in Parkinson's disease as their precursors could be an alternative source for neurons for transplantation or recruitment to lesion sites (Lie et al., 2004). Lineage analyses showed that these dopaminergic neurons mostly originate from the dorsal PVZ. At the molecular level, several transcription factors were shown to be required for proper differentiation of these dopaminergic neurons, namely Pax6, Dlx2, Id2, Klf7, and ER81 (Hack et al., 2005; Kohwi et al., 2005; Saino-Saito et al., 2007; Brill et al., 2008; Havrda et al., 2008; Caiazzo et al., 2011). Among these factors, Pax6 and Dlx2 seem to play a major role as their overexpression was able to increase the generation of this type of neurons (Hack et al., 2005; Kohwi et al., 2005; Brill et al., 2008). However, additional expression and functional data draw a more complex situation that hardly fits with the specific involvement of these two TFs in the specification of the dopaminergic phenotype in the OB. Indeed, it has been suggested that Pax6 is not confined to the dorsal PVZ, but widely expressed in most RMS neuronal precursors where it is required for induction of neuronal commitment in general (Hack et al., 2005). In contrast, Dlx2 mRNA expression seems to be restricted to the lateral PVZ (Brill et al., 2008), which is in contradiction with the dorsal origin of OB dopaminergic neurons (Merkle et al., 2007).

Here we provide detailed and dynamic expression data that offer a more detailed picture and reconcile these discrepancies. First, we show that Pax6 protein expression is not distributed as widely as expected, but merely restricted to the dorsal lineage from the PVZ stem cells to the mature neurons of the OB. Second, we show that while Dlx2 is initially restricted to lateral progenitors, its expression is at later time points also induced in descendants of the dorsal PVZ, thus, explaining how Dlx2 can regulate dopaminergic fate in this system. 


\section{MATERIALS AND METHODS ANIMALS}

All animals were treated according to protocols approved by the French Ethical Committee. CD1 mice (Charles River, Lyon, France) were used for in vivo electroporation and expression pattern analyses.

\section{FOREBRAIN ELECTROPORATION}

P1 pups were electroporated as described in (Boutin et al., 2008). $2 \mu \mathrm{l}$ of a pCAGGS-eGFP plasmid solution at a concentration of $5 \mu \mathrm{g} / \mu \mathrm{l}$ in PBS was injected into the brain LV. Electroporation was performed using the CUY21 edit device and the $10 \mathrm{~mm}$ tweezers electrodes (CUY650P10) (Nepagene, Chiba, Japan) coated with conductive gel (Signagel, Parker Laboratories, USA). Electrodes were distinctly positioned in order to target either the lateral or the dorsal wall of the ventricle.

\section{IMMUNOHISTOCHEMISTRY AND IMAGE ANALYSES}

Immunohistochemistry was performed on free-floating sections as described previously (Boutin et al., 2010). Optical images were taken using a fluorescence microscope (Axiolmager Z1, ApoTome system, Zeiss, Germany) or a laser confocal scanning microscope (LSM510, Zeiss, Germany).

Primary antibodies: anti-Pax6 (mouse from Millipore, 1:700; rabbit from Covance, 1:400), the anti-Dlx2 was provided by Prof. Yoshikawa. Secondary antibodies include anti-rabbit HRP conjugated (Jackson ImmunoResearch Laboratories), anti-mouse, anti-rabbit and anti-chicken Alexa 555 conjugated (Invitrogen). All images were analyzed blind to the experimental condition. Data are presented as mean \pm SEM. Unless otherwise specified, $n$ represents the number of animals analyzed. Two-tailed unpaired Student's $t$-test was used to assess differences between data groups using Instat software (Graph Pad Software). Differences were considered statistically significant when $P<0.05$.

\section{MICROARRAY ANALYSIS}

For sorting GFP fluorescent cells, pups are sacrificed at different times after in vivo electroporation and brain tissue from which cells have to be sorted are dissected under the microscope. Cells are dissociated as described before (Seidenfaden et al., 2006) and fluorescent cells were sorted using a MoFlo flow cytometry sorter (Beckman Coulter). RNA was extracted, amplified using the SuperAmp Technology (Miltenyi), and hybridized (two colors protocol, each color corresponding to a targeted region, dorsal or lateral wall) on a G4122F Whole Mouse Genome $(4 \times 44 \mathrm{~K})$ Oligo Microarrays (Agilent) as described previously (Boutin et al., 2010). Results were processed as described previously (Boutin et al., 2010). For conditions 2 ( 8 hpe and 2 dpe) or 3 (1, 4, 7 , $14 \mathrm{dpe})$ independent repetitions were performed and data are presented as mean \pm SEM of normalized intensity values.

\section{RESULTS}

\section{Pax6 PROTEIN EXHIBITS A DORSAL EXPRESSION IN THE PVZ}

Immunohistochemistry showed that most of the PVZ cells expressing Pax6 are located in the dorsal wall of the ventricle (Figure 1A). In the lateral wall Pax6 expression exhibits a dorsoventral descending gradient leading to the total absence of Pax6 detection in the most ventral part of the PVZ (Figure 1A). To analyze the time course and extent of Pax6 expression in the dorsal PVZ we used in vivo electroporation. This technique presents two main advantages. First, it allows the specific transfection of radial glia (Doetsch et al., 1999; Boutin et al., 2008, 2010), the primary NSCs in the system. Second, it is possible to target specific PVZ regions, such as dorsal vs. lateral walls, through the orientation of the electrical field applied to the mouse head (Fernandez et al., 2011) (Figure 1B). Therefore, this technique allows discriminating dorsally vs. laterally generated cells in the RMS and $\mathrm{OB}$, although these cells are intermingled in both structures.

We used expression of GFP to label cohorts of cells in the system and isolate these cohorts at different time points. It has been shown that 6 hours post-electroporation (hpe) of a GFP-expressing plasmid in the PVZ green cells are radial glial NSCs, while at 2 days post-electroporation (dpe) they are a mix of NSCs, transit-amplifying precursors and neuroblasts (Boutin et al., 2008). At 4 dpe in RMS the $\mathrm{GFP}^{+}$cells are migrating neuroblasts and at 15 dpe in the $\mathrm{OB}$ they are differentiating neurons (Boutin et al., 2008, 2010).

When electroporated the dorsal wall of P1 mice we observed that at 6 hpe more than $80 \%$ of the electroporated radial glial cells expressed Pax6 endogenously (Figures 1C,E). The ratio remained similar two days later in transit-amplifying and neuroblast precursors $\left(80 \pm 5 \% \mathrm{Pax}^{+} / \mathrm{GFP}^{+}\right.$cells; Figures 1D,E). In contrast, the percentage of $\mathrm{GFP}^{+}$cells expressing Pax6 dropped to around $20 \%$ in a region comprising $100 \mu \mathrm{m}$ of the most dorsal part of the lateral PVZ (the horn in Figure 1A) and became almost null in the most ventral PVZ $\left(3 \pm 1 \% \mathrm{Pax}^{+} / \mathrm{GFP}^{+}\right.$cells in lateral PVZ; Figures 1C-E) at both time points ( $n=3$ animals per group, more than 200 cells per group, $\left.{ }^{*} p<0.05,{ }^{* *} p<0.01\right)$. These results, showing a restriction of Pax6 protein to the dorsal PVZ, are in perfect agreement with a specific involvement of Pax6 in dopaminergic fate determination. The fact that the primary NSCs already differentially express Pax6 in a dorso-lateral gradient suggests that this determination occurs precociously in the immature NSCs.

\section{Pax6 EXPRESSION IN NEUROBLASTS AND DIFFERENTIATED NEURONS IS PREFERENTIALLY FOUND IN THE DORSAL PVZ LINEAGE}

Several previous data suggested a requirement of Pax6 for general OB neurogenesis (Hack et al., 2004, 2005; Jang and Goldman, 2011). This statement does not fit with our data showing a restriction of Pax6 expression in the dorsal aspect of the PVZ but might be based on an expression in the lateral lineage at later time points in the RMS or OB. We thus investigated Pax6 expression in the lateral lineage in comparison to the dorsal lineage to determine whether Pax6 expression is activated in lateral descendents at later differentiation stages, i.e., at 4 dpe in the RMS and at 15 dpe in the OB.

Observation of electroporated cells at 4 dpe confirmed that migrating RMS neuroblasts are intermingled (Figure 2A). In the $\mathrm{OB}$ at 15 dpe, dorsally electroporated cells were mostly superficial granule and periglomerular neurons while laterally electroporated cells colonized the deep layers of the granule cell layer (GCL) (Figure 2B). Four dpe, the percentage of $\mathrm{GFP}^{+}$cells expressing Pax6 in the RMS remained significantly higher in the dorsal 

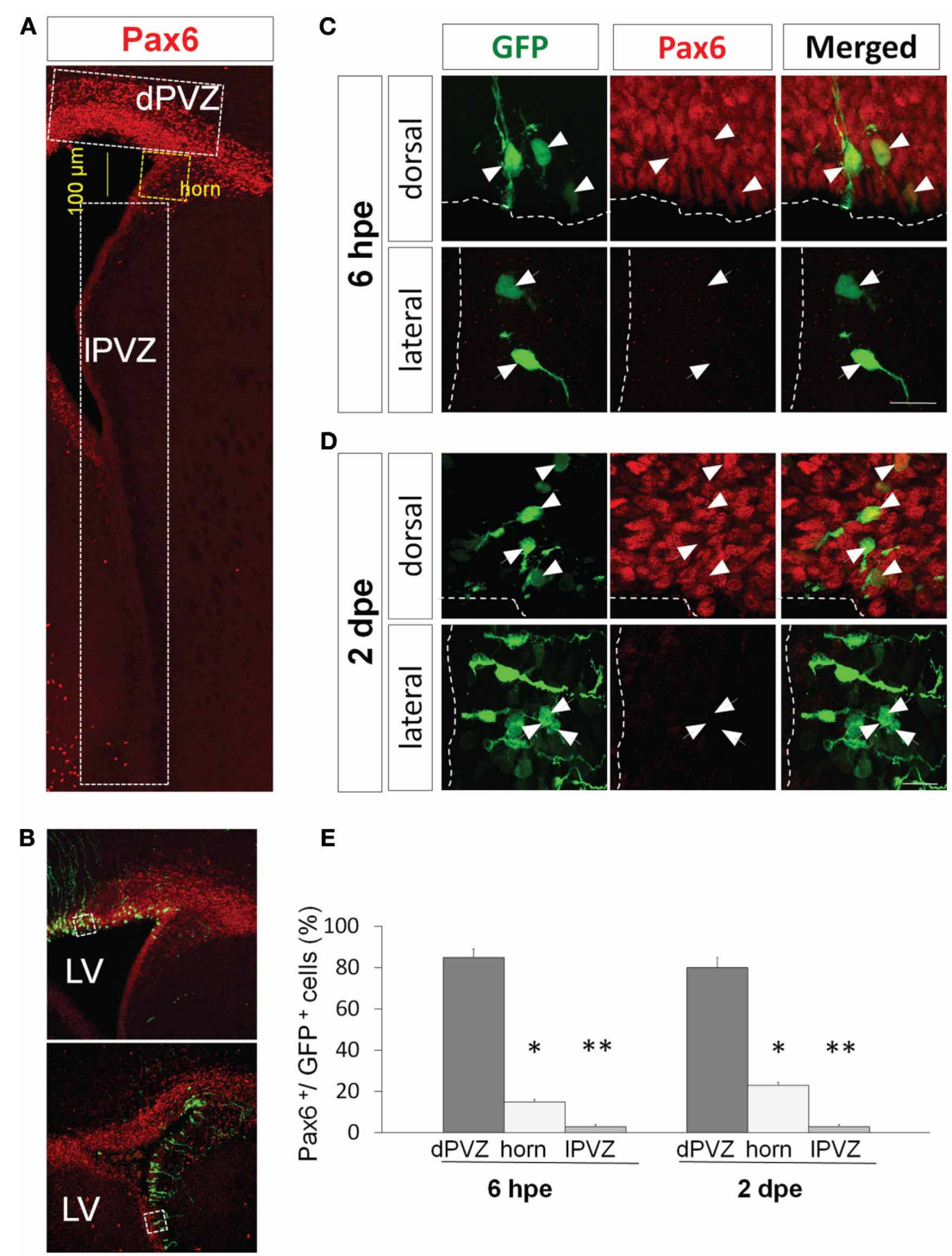

FIGURE 1 | Pax6 expression is restricted dorsally in the periventricular region. (A) Low magnification view of a coronal section of the P1 forebrain labeled with Pax6 antibody (red). The signal is strong and widespread in the dorsal periventricular zone (dPVZ), weaker at the junction between the dorsal and lateral walls (horn and yellow) and faint and rare in the lateral wall (IPVZ).

(B) PVZ coronal section $6 \mathrm{~h}$ after a dorsal (top) or a lateral (bottom) electroporation with a GFP expressing plasmid. Pax6 is labeled in red. (C and D) Pax6 expression (red) by GFP-expressing cells after GFP electroporation in the dorsal (upper panel) or lateral wall (lower panel).
At 6 hpe (C), radial glial neural stem cells express Pax6 in the dorsal, but not lateral, PVZ. (D) Similarly, most amplifying precursors and neuroblasts of the dorsal but not lateral PVZ express Pax6 (arrowheads: $\mathrm{GFP}^{+} / \mathrm{Pax}^{+}$cells, arrows: $\mathrm{GFP}^{+} / \mathrm{Pax}^{-}$cells). (E) Histograms depicting in three regions of the PVZ (dorsal PVZ, horn, and lateral PVZ) the percentage of GFP electroporated cells co-expressing Pax 6 h or $2 \mathrm{~h}$ days after dorsal or lateral electroporation ( $n=3$ animals per group, more than 200 cells per group, $\left.{ }^{*} p<0.05,{ }^{* *} p<0.01\right)$. LV: lateral ventricle. Scale bars: $20 \mu \mathrm{m}$. than in the lateral lineage $\left(49 \pm 8 \%\right.$ vs. $11 \pm 1 \% \mathrm{Pax}^{+} / \mathrm{GFP}^{+}$ cells, respectively; $n=3-5$ mice, more than 100 cells per group, $p<0.05$ ) (Figures 2C,D). At 15 dpe, we determined the ratio of $\mathrm{GFP}^{+}$cells expressing Pax6 in the granule cell and the glomerular layers (GCL and GL, respectively), the two target layers of OB neurogenesis. In both dorsal and lateral electroporations, the proportion of $\mathrm{GFP}^{+}$cells expressing Pax6 was significantly higher in the GL than in the GCL. This was in accordance with the role of Pax6 in the determination of dopaminergic neurons that are confined to the GL. Importantly, we again observed a strong preferential expression of Pax6 in cells issued from the dorsal PVZ in both GCL and GL $\left(48 \pm 5 \%\right.$ vs. $17 \pm 2 \% \mathrm{Pax}^{+} / \mathrm{GFP}^{+}$ cells in GCL and $97 \pm 2 \%$ vs. $56 \pm 5 \% \mathrm{Pax}^{+} / \mathrm{GFP}^{+}$cells in GL; Figures 2C,D).

Altogether these data show that, in accordance with its role in dopaminergic fate determination, Pax6 expression is preferentially expressed in the entire dorsal lineage throughout the neurogenic process. 

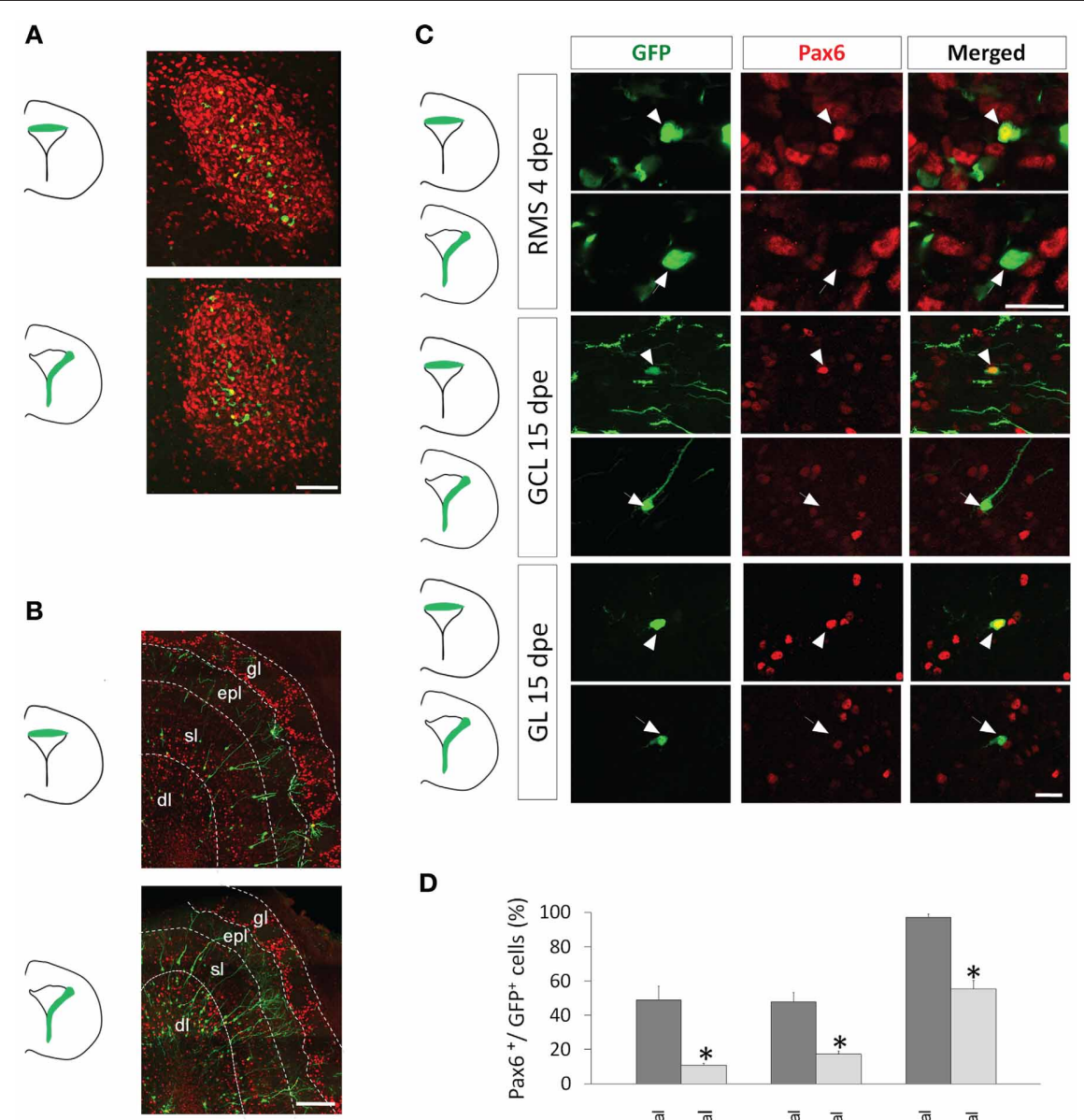

D

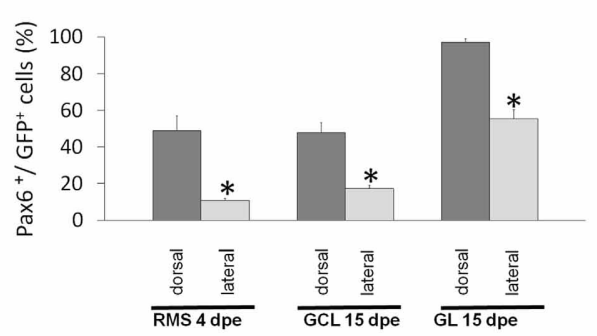

FIGURE 2 | Pax6 remains preferentially expressed in the dorsal lineage in neuroblasts migrating in the RMS and in new neurons of the olfactory bulb. (A and B) Distribution of dorsally or laterally generated GFP+ cells in the RMS at 4 dpe (A) and in the olfactory bulb at $15 \mathrm{dpe}$ (gl: glomerular layer, epl: external plexiform layer, sl: superficial layer of the granule cell layer, dl: deep layer of the granule cell layer) (B). (C) 3-D reconstructions of merged images of dorsally (upper panel) or laterally (lower panel) electroporated GFP+ cells (green) labeled with Pax6 antibody (red) at 4 or 15 dpe. These images show that dorsally but not laterally generated $\mathrm{GFP}^{+}$cells in the RMS and in the granule cell layer (GCL) or glomerular layer (GL) of the OB express Pax6. (D) Histograms representing the percentage of GFP electroporated cells expressing Pax6 4 days after electroporation in the RMS and 15 days after electroporation either in the GCL or in the GL, after dorsal (dark gray bars) or lateral (light gray bars) electroporation ( $n=3-5$ mice per group). Scale bars: A, $50 \mu \mathrm{m}$; B, $200 \mu \mathrm{m}$; C, $20 \mu \mathrm{m}$.

\section{DIX2 EXPRESSION IS EXCLUSIVE TO THE LATERAL PVZ BUT IS ACTIVATED IN ALL RMS NEUROBLASTS}

A function for Dlx2 in dopaminergic subtype specification during OB neurogenesis has been proposed (Brill et al., 2008). However, in situ hybridization data showed mRNA expression only in the lateral PVZ (Brill et al., 2008), which is in disagreement with a role of Dlx2 in the dopaminergic fate specification, since dopaminergic neurons arise mostly from the dorsal PVZ (Merkle et al., 2007). We thus revisited the expression of Dlx 2 in the entire dorsal and lateral lineages by immunohistochemistry.

In PVZ, we detected Dlx2 protein almost exclusively in the lateral aspect, confirming the available in situ hybridization data (Brill et al., 2008) (Figure 3A). We then electroporated a GFP plasmid in the lateral and dorsal PVZ and searched for Dlx2 expression in the electroporated cells. We observed that 6 hpe all fluorescent radial glia cells were Dlx2 negative (Figures 3B,C, and $4 \mathrm{E})$. However, Dlx2 protein was expressed in most $\mathrm{GFP}^{+}$ transit-amplifying cells and neuroblasts derived from lateral radial glia two days after electroporation ( $93 \pm 1 \%$; Figures $3 E$ and $4 \mathrm{E})$. In contrast, barely any dorsally electroporated radial glia or immediate PVZ descendants expressed Dlx2 (2 $\pm 1 \%$; Figures 3B,D, and 4E; $n=3-5$ mice, more than 100 cells per group; ${ }^{*} p<0.05,{ }^{* *} p<0.01$, and $\left.{ }^{* * *} p<0.001\right)$ despite the occasional occurrence of other cells that expressed Dlx2 at low level (Figures 3B,D). These results show that in the PVZ, Dlx2 is restricted to lateral transit-amplifying cells and neuroblasts and that Pax6 and Dlx2 expressions are not overlapping in PVZ stem cells and early progenitors.

Then, we investigated the time course of Dlx2 expression at later stages to determine whether Dlx2 expression is induced later 

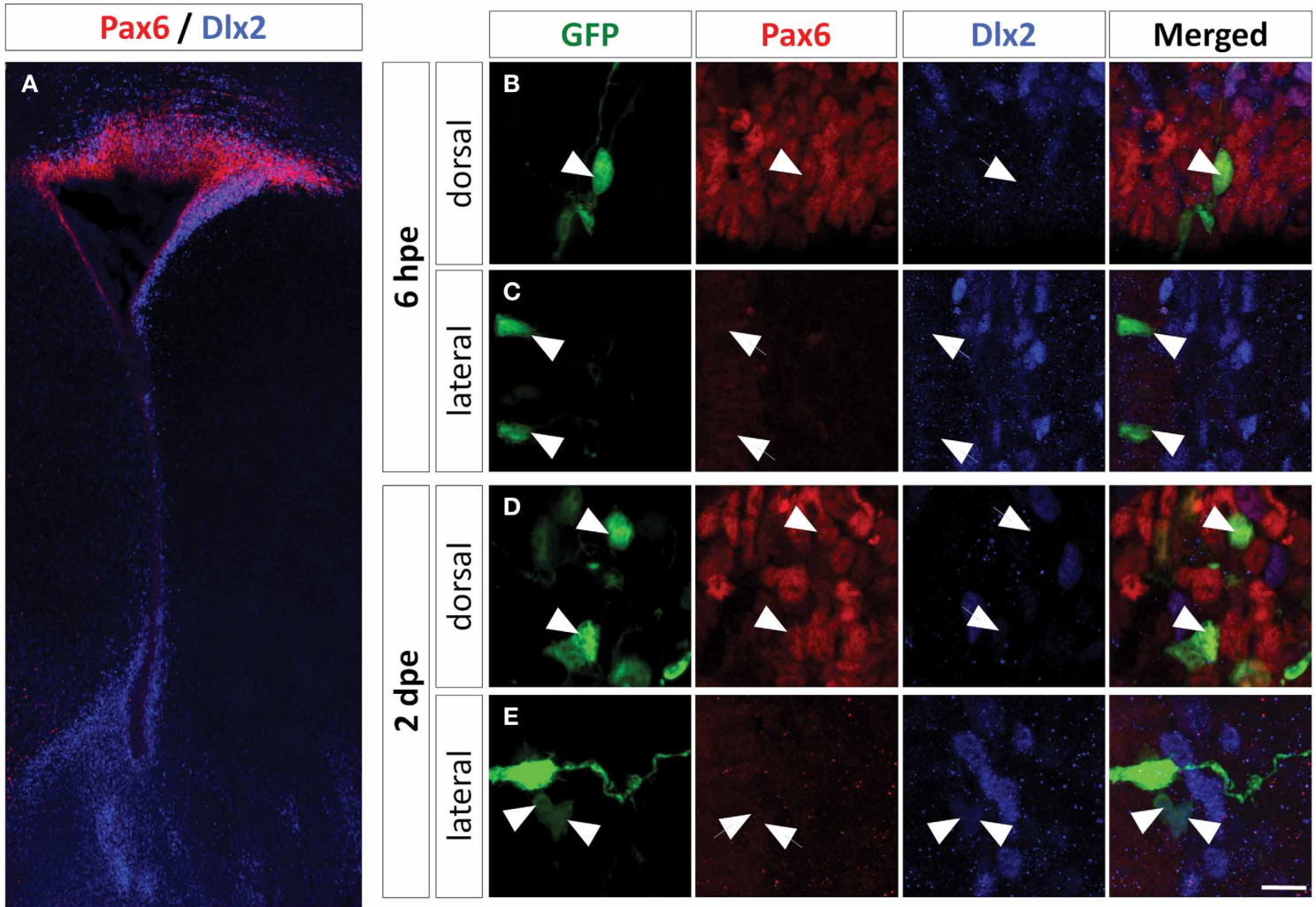

FIGURE 3 | DIx2 expression in the PVZ is restricted to the non-radial glial progenitor cells of the lateral wall. (A) Low magnification of a P1 mouse brain coronal section labeled with Pax6 (red) and DIx2 (blue) antibodies showing an overall complementary, non-overlapping expression of these two genes in the PVZ. (B-E) High magnification of dorsally or laterally GFP electroporated cells labeled with Pax6 and Dlx2 antibodies at $6 \mathrm{~h}(\mathbf{B}, \mathbf{C})$ and two days $(\mathbf{D}, \mathbf{E})$ after electroporation. Note that the dorsally electroporated cells expressed Pax6 but not DIx2 (B,D). As expected the laterally electroporated cells did not express Pax6 at any time. The lateral radial glial neural stem cells did not express DIx2 at 6 hpe (C), while the transit-amplifying and neuroblast progeny did express Dlx2 at 2 dpe (E). Arrowheads: $\mathrm{GFP}^{+} / \mathrm{marker}^{+}$cells, arrows: GFP ${ }^{+} /$marker $^{-}$cells. Scale bar: $10 \mu \mathrm{m}$. in the dorsal-derived population. We first electroporated a GFP plasmid either dorsally or laterally in the PVZ and then isolated $\mathrm{GFP}^{+}$cells by Fluorescent Activated Cell Sorting (FACS) at different time points. Genome-wide transcriptomic microarrays were then performed on amplified material from sorted cells. At 8 hpe, a stage at which the sorted cells were predominantly radial glia, Dlx2 intensity values were low in all samples. In contrast, at 1 and 2 dpe Dlx2 expression was significantly increased in the lateral samples but remained low in the dorsal fraction (Figure 4A). These results are in full agreement with our immunohistochemical analyses (Figures 3B-E). Interestingly, we observed an activation of Dlx2 expression at 4,7 , and 14 dpe in the dorsally electroporated cells, in both RMS and OB. Meanwhile, Dlx2 expression was maintained high in the laterally electroporated cells (Figure 4A).

To further confirm these data we performed immunohistochemical analyses on GFP electroporated cells at 4 and 15 dpe using Dlx2 and Pax6 antibodies. The results confirmed the microarray data, showing that in contrast to 6 hpe and 2 dpe the majority of dorsally electroporated RMS neuroblasts and OB neurons induced Dlx2 expression in addition to Pax6 at 4 and 15 dpe, respectively, (96 $\pm 1 \% \mathrm{Dlx} 2^{+} / \mathrm{GFP}^{+}$cells in RMS vs. $97 \pm 3 \%$ in $\mathrm{OB}$, Figures 4B-D). As expected, most laterally electroporated cells remained Dlx2 ${ }^{+}$at these time points $\left(90 \pm 3 \% \mathrm{Dlx} 2^{+} / \mathrm{GFP}^{+}\right.$ cells in RMS vs. $98 \pm 2 \%$ in OB; Figures $4 \mathrm{E}$ and 5 ).

In conclusion, this demonstrates that while lateral cells are Dlx2-positive from early time points in the PVZ, dorsally derived neuroblasts induce Dlx2 at later time points, during their journey to the $\mathrm{OB}$

\section{DISCUSSION}

Cell replacement for the treatment of neurodegenerative diseases may be achieved either by grafting neurons or by mobilizing ongoing postnatal neurogenesis (Lie et al., 2004). In this view, OB neurogenesis presents some evident advantages, especially in the context of Parkinson's disease as it generates dopaminergic neurons that survive this condition (Hoglinger et al., 2004; Winner et al., 2006). OB neurogenesis may be a natural source for neuronal replacement that could circumvent the ethical, provisioning, and immune rejection problems brought by transplantations (Isacson, 2003). Furthermore, from a fundamental point of view, $\mathrm{OB}$ neurogenesis is a very amenable system that 


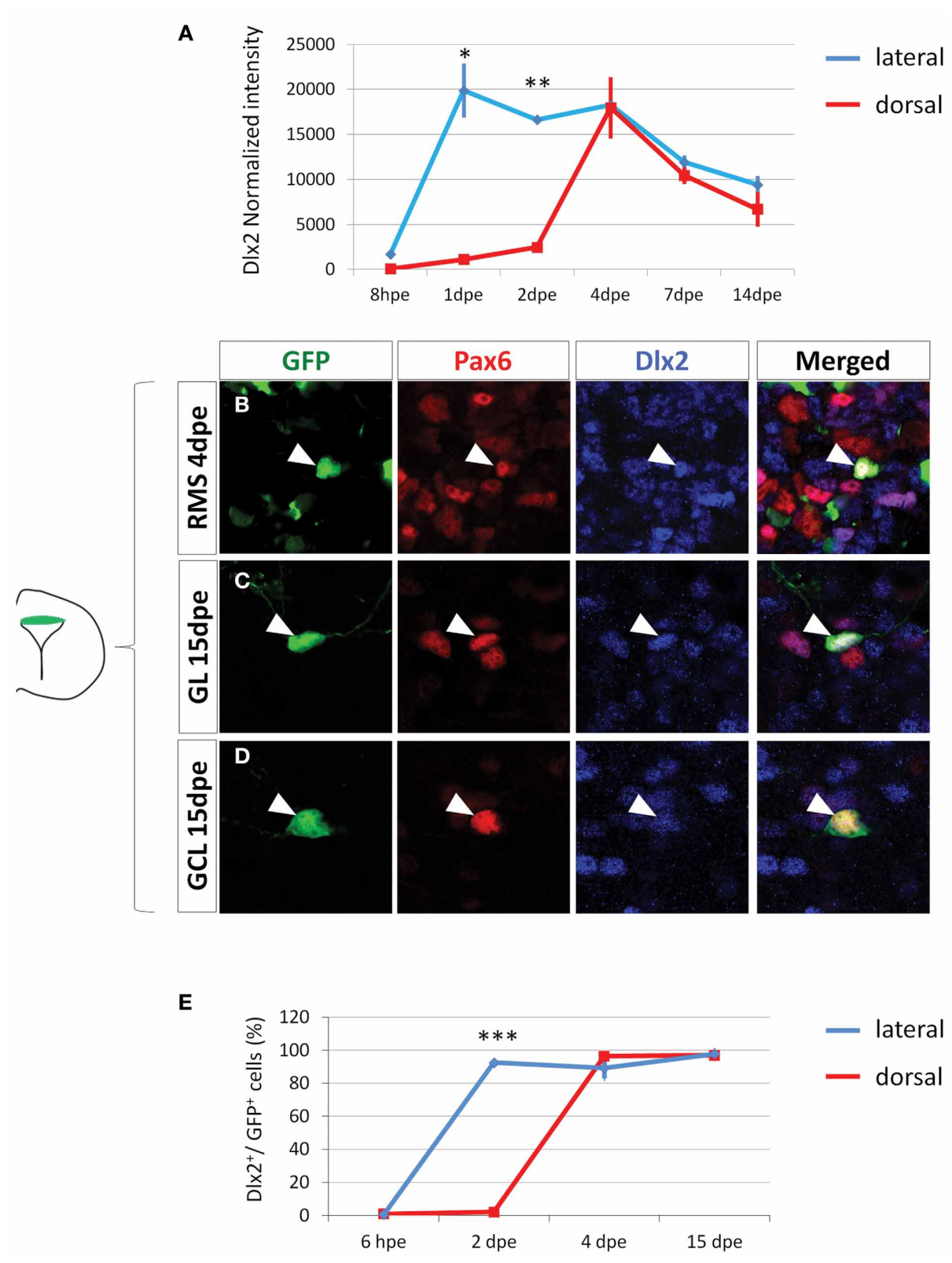

FIGURE 4 | DIx2 gets activated in the dorsal lineage once cells have entered the RMS and is maintained in the OB. (A) Evolution of Dlx2 transcript expression over time in the dorsal (red) and lateral (blue) lineages obtained from microarray analysis performed on FAC-sorted GFP electroporated cells at successive time points. (B-D) High

magnifications of dorsally generated GFP ${ }^{+}$cells labeled with Pax6 (red) and DIx2 (blue) antibodies in the RMS at 4 dpe (B), or in the granule cell layer (C) and glomerular layer (D) at $15 \mathrm{dpe}$. Arrowheads: $\mathrm{GFP}^{+} /$marker $^{+}$cells. Note that both in the RMS and the GCL and GL of the OB Dlx2 is co-expressed with Pax6 in dorsally generated GFP electroporated cells. (E) Evolution of DIx2 expression in dorsal (red) or lateral (blue) $\mathrm{GFP}^{+}$electroporated cells at successive time after electroporation ( $n=3$ mice per group). Scale bar: $10 \mu \mathrm{m}$. allows studying the control of neurogenesis in general and also the molecular basis of neuronal diversity. The system is of particularly suited to investigate how the dopaminergic fate in the forebrain is determined.

Previous work has established the transcription factors Pax6 and Dlx2 as regulators of the dopaminergic specification during postnatal $\mathrm{OB}$ neurogenesis, together with few other factors (Havrda et al., 2008). Pax6 appears to play a major role in this specification as Pax6 mutant PVZ precursors grafted to a host PVZ are able to generate OB neurons except for the dopaminergic subtype (Kohwi et al., 2005). Furthermore, overexpression or inhibition of Pax6 by retroviral injections in vivo increases and reduces the proportion of $\mathrm{OB}$ dopaminergic neurons in the $\mathrm{OB}$, respectively (Hack et al., 2005). The function of Dlx2 in the system is less specific as lack of Dlx2 interferes with neuron production in general (Qiu et al., 1995). Nevertheless, Dlx2 can induce 


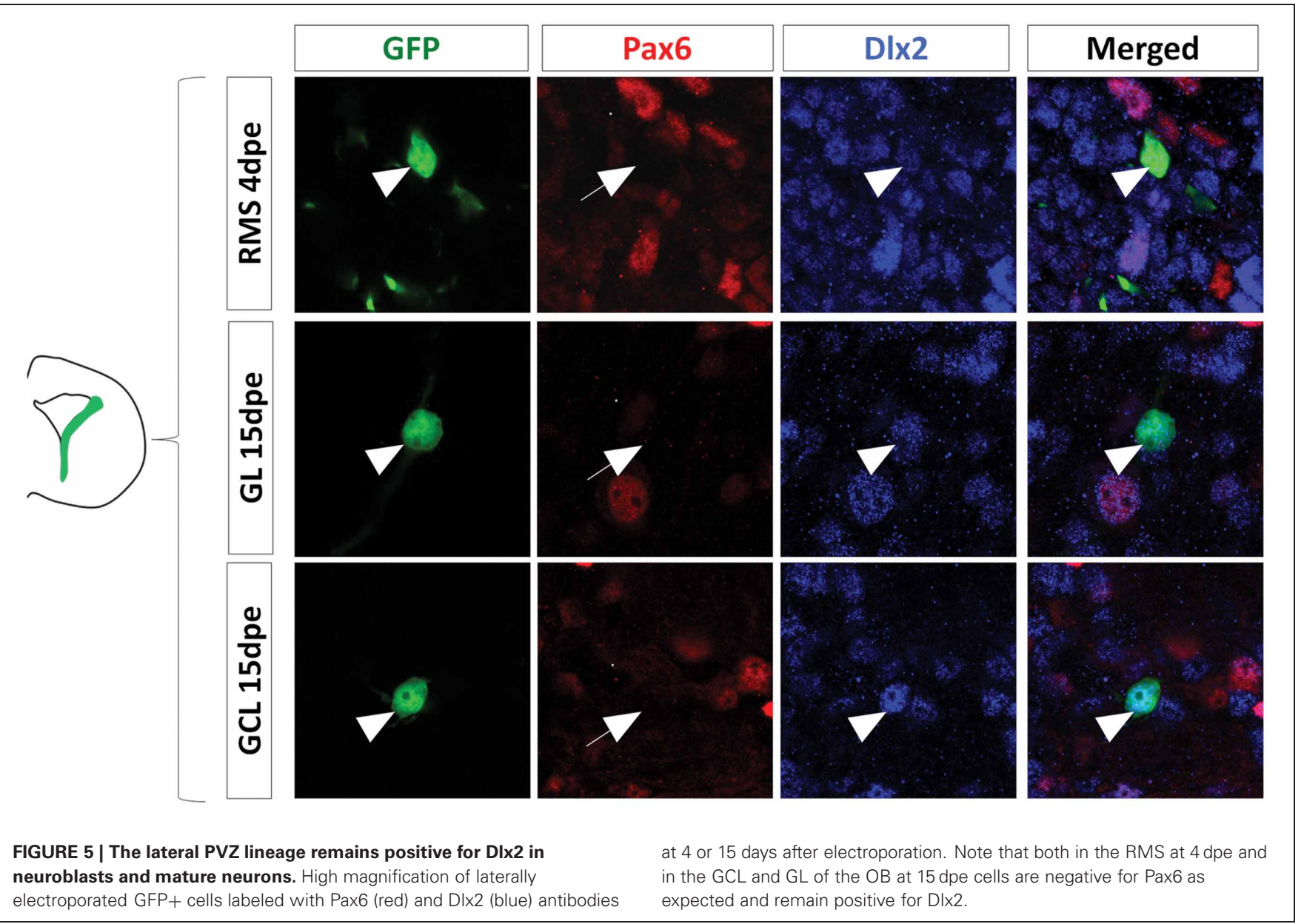

the dopaminergic fate in this system because its overexpression in the PVZ increases the genesis of $\mathrm{OB}$ dopaminergic neurons (Brill et al., 2008).

Surprisingly these functional analyses were not fully supported by several expression data. Indeed, Pax6 expression studies indicated that most, if not all, neuroblasts express Pax6 in the RMS (Hack et al., 2005). This, plus the fact that Pax6 induces neurons in neurosphere cells that otherwise generate predominantly glia (Hack et al., 2004) lead these authors to postulate that Pax6 is a general neurogenic factor for OB neurogenesis (Hack et al., 2005).

We show here, using a technique allowing neuronal lineage tracing at both the regional and cellular levels that Pax6 is in fact highly enriched in the dorsally generated cells, from the stem cell in PVZ to the differentiated neuron in the OB. Pax6 is only very rarely found in a laterally generated cell. This high resolution expression analyses show that Pax6 is not required for lineage progression of laterally generated cells, indicating that the transcription factor does not act as a general neurogenic factor. Instead, a major role of Pax6 in this system appears to be the determination of the dopaminergic neurotransmitter phenotype in the GL (Hack et al., 2005; Kohwi et al., 2005). However, the existence of a substantial proportion of GCL neurons expressing Pax6 (Figure 2D) suggests additional roles for Pax6 in OB neurogenesis.
Another paradox concerns the apparent discrepancy between Dlx2 expression and its role in dopaminergic specification. Indeed, Dlx2 mRNA appears restricted to the lateral PVZ (Brill et al., 2008) whereas dopaminergic neurons are generated by dorsal PVZ (Merkle et al., 2007). Using in vivo electroporation, dynamic microarray analyses, and immunohistochemistry, we show that Dlx2 expression in dorsally generated cells is switched on in RMS neuroblasts and maintained in the OB. These data fit with the functional data stating a dual role of Dlx2 in the neurogenesis in general and a more specific involvement in the dopaminergic fate specification (Brill et al., 2008). Dlx2 expression is temporally regulated in the process. Indeed, at the PVZ transit-amplifying cells stage Dlx2 is confined to the lateral lineage but absent from dorsal cells. However, once in the RMS as the transcription factor is induced in migrating neuroblasts of both lineages. Whether this complex regulation of expression timing has functional significance needs to be investigated.

In conclusion, we present here dynamic expression data for two genes showing a regionalized expression in the PVZ. This regionalized expression for Pax6 reveals a specific and permanent involvement in dopaminergic fate determination. For Dlx2 however, this regionalized expression is transient in accordance with the more general role of this gene in neurogenesis. We believe that our study highlights the necessity, when studying molecular 
determinants of fate specification, to follow their expression in a dynamic fashion as performed in our comparative genomic screen on dorsally vs. laterally electroporated cells at different times after electroporation of the primary stem cells. Extending this analysis to the whole genome will draw a detailed spatial and temporal expression map of important genes in postnatal OB neurogenesis. Similarly to what we describe here for Pax6 and Dlx2 this map might allow us to validate, reject or even identify new molecular determinants for neuronal subtype specification in the postnatal brain.

\section{REFERENCES}

Alvarez-Buylla, A., and GarciaVerdugo, J. M. (2002). Neurogenesis in adult subventricular zone. $J$. Neurosci. 22, 629-634.

Boutin, C., Diestel, S., Desoeuvre, A., Tiveron, M. C., and Cremer, $\mathrm{H}$. (2008). Efficient in vivo electroporation of the postnatal rodent forebrain. PLoS One 3, e1883. doi: 10.1371/journal.pone.0001883

Boutin, C., Hardt, O., de Chevigny, A., Core, N., Goebbels, S., Seidenfaden, R., Bosio, A., and Cremer, H. (2010). NeuroD1 induces terminal neuronal differentiation in olfactory neurogenesis. Proc. Natl. Acad. Sci. U.S.A. 107, 1201-1206.

Brill, M. S., Snapyan, M., Wohlfrom, H., Ninkovic, J., Jawerka, M., Mastick, G. S., Ashery-Padan, R., Saghatelyan, A., Berninger, B., and Gotz, M. (2008). A dlx2- and pax6-dependent transcriptional code for periglomerular neuron specification in the adult olfactory bulb. J. Neurosci. 28, 6439-6452.

Caiazzo, M., Colucci-D'Amato, L., Volpicelli, F., Speranza, L., Petrone, C., Pastore, L., Stifani, S., Ramirez, F., Bellenchi, G. C., and di Porzio, U. (2011). Kruppel-like factor 7 is required for olfactory bulb dopaminergic neuron development. Exp. Cell Res. 317, 464-473.

Doetsch, F., Caille, I., Lim, D. A., Garcia-Verdugo, J. M., and AlvarezBuylla, A. (1999). Subventricular zone astrocytes are neural stem cells in the adult mammalian brain. Cell 97, 703-716.

Fernandez, M. E., Croce, S., Boutin, C., Cremer, H., and Raineteau, O. (2011). Targeted electroporation of defined lateral ventricular walls: a novel and rapid method to study

\section{ACKNOWLEDGMENTS}

We thank Aude Barani from the Precym platform of Centre d'Océanologie de Marseille for FACS and Xavier Morin for plasmids. This work was supported by funds to Harold Cremer from the Agence National de la Recherche (ANR, FORDOPA), Foundation pour la Recherche Médicale (Label Equipe FRM), and the European Commission (MarieCurie ITN AXREGEN and IAPP "DopaNew") and by funds to Andreas Bosio from the European Commission (IAPP "DopaNew").

progenitors of the neonatal subventricular zone. PLoS One 6, e20894. doi: 10.1371/journal.pone.0020894

Kohwi, M., Osumi, N., Rubenstein, J. L., and Alvarez-Buylla, A. (2005). Pax6 is required for making specific subpopulations of granule and periglomerular neurons in the olfactory bulb. J. Neurosci. 25, 6997-7003.

Lie, D. C., Song, H., Colamarino, S. A., Ming, G. L., and Gage, F. H. (2004). Neurogenesis in the adult brain: new strategies for central nervous system diseases. Annu. Rev. Pharmacol. Toxicol. 44, 399-421.

Lledo, P. M., Alonso, M., and Grubb, M. S. (2006). Adult neurogenesis and functional plasticity in neuronal circuits. Nat. Rev. Neurosci. 7 , 179-193.

Lledo, P. M., Merkle, F. T., and AlvarezBuylla, A. (2008). Origin and function of olfactory bulb interneuron diversity. Trends Neurosci. 31, 392-400.

Merkle, F. T., Mirzadeh, Z., and Alvarez-Buylla, A. (2007). Mosaic organization of neural stem cells in the adult brain. Science 317 , 381-384.

Qiu, M., Bulfone, A., Martinez, S. Meneses, J. J., Shimamura, K., Pedersen, R. A., and Rubenstein, J. L. (1995). Null mutation of Dlx-2 results in abnormal morphogenesis of proximal first and second branchial arch derivatives and abnormal differentiation in the forebrain. Genes Dev. 9, 2523-2538.

Saino-Saito, S., Cave, J. W., Akiba, Y., Sasaki, H., Goto, K., Kobayashi, K., Berlin, R., and Baker, H. (2007). ER81 and CaMKIV identify anatomically and phenotypically defined subsets of mouse olfactory bulb interneurons. J. Comp. Neurol. 502, 485-496.

Seidenfaden, R., Desoeuvre, A., Bosio, A., Virard, I., and Cremer, $\mathrm{H}$. (2006). Glial conversion of SVZderived committed neuronal precursors after ectopic grafting into the adult brain. Mol. Cell. Neurosci. 32, 187-198.

Winner, B., Geyer, M., CouillardDespres, S., Aigner, R., Bogdahn, U., Aigner, L., Kuhn, G., and Winkler, J. (2006). Striatal deafferentation increases dopaminergic neurogenesis in the adult olfactory bulb. Exp. Neurol. 197, 113-121.

Conflict of Interest Statement: The authors declare that the research was conducted in the absence of any commercial or financial relationships that could be construed as a potential conflict of interest.

Received: 16 December 2011; paper pending published: 05 January 2012; accepted: 06 February 2012; published online: 27 February 2012.

Citation: de Chevigny A, Core $N$, Follert P, Wild S, Bosio A, Yoshikawa K, Cremer H and Beclin C (2012) Dynamic expression of the pro-dopaminergic transcription factors Pax6 and Dlx2 during postnatal olfactory bulb neurogenesis. Front. Cell. Neurosci. 6:6. doi: 10.3389/fncel.2012.00006

Copyright (C) 2012 de Chevigny, Core, Follert, Wild, Bosio, Yoshikawa, Cremer and Beclin. This is an open-access article distributed under the terms of the Creative Commons Attribution Non Commercial License, which permits non-commercial use, distribution, and reproduction in other forums, provided the original authors and source are credited. 